\title{
Supervivencia de cáncer de mama tratado con quimioterapia neoadyuvante
}

\author{
Survival of breast cancer treated with neoadjuvant chemotherapy
}

- Pedro Luis Ramos Guette1, María Atenas Ramos Escalante², Diana Silva³, Mario Gonzales4, Mauricio García

' Médico, Especialista en Medicina Interna, Especialista en Oncología Clínica, Epidemiólogo Clínico, Universidad Nacional de Colombia. 2 Estudiante de Medicina, Universidad del Rosario.

Jefe enfermera de Oncología, Pontificia Universidad Javeriana, Instituto Nacional de Cancerología, Universidad Nacional de Colombia.

(nstituto Nacional de Cancerologí, Universidad Nacional de Colombia.

5 Médico, Especialista en Cirugía Oncológica, Pontificia Universidad Javeriana, Instituto Nacional de Cancerología, Universidad Nacional de Colombia.

\section{Resumen}

Objetivo: evaluar la sobrevida global y libre de enfermedad en mujeres con cáncer de mama que reciben tratamiento de quimioterapia neoadyuvante en la clínica Oncocare durante el período de 2005 a 2014.

Métodos: este estudio involucró el análisis de información en el período comprendido entre el $1^{\circ}$ de enero de 2005 y el 31 diciembre de 2014. Se incluyeron todas las pacientes de 18 años o más con diagnóstico de cáncer de mama que reciben tratamiento de quimioterapia neoadyuvante. Se construyeron curvas de supervivencia por el método de Kaplan-Meier, se compararon con el método de rangos logaritmos y se realizó un análisis de regresión de rangos proporcionales por Cox.

Resultados: 350 pacientes fueron identificadas en el estudio. La edad media fue de 54,8 años. El estadio clínico más frecuente según versión AJCC 7a edición de 2009 fue IIIB 125 (35,71\%), seguido por IIIA 96 (27,43\%) y IIIC 58 (16,57\%). Los receptores de estrógenos son positivos en un $67,14 \%$, los de progestágeno son positivos en un $54,29 \%$ y los receptores de HER2 son positivos en un 15,43\%; durante el seguimiento se observaron 81 (23,14\%) eventos de recaída y 70 (20\%) eventos de muerte.

Los tiempos de sobrevida libre de enfermedad se ven disminuidos por el compromiso de 10 o más ganglios HR 2,96 (IC95\% 1,24-3,27) y se aumentan en respuestas patológicas completas HR 0,29 (IC95\% 0,11-0,72).

Los tiempos de sobrevida global se ven reducidos por el compromiso de 10 o más ganglios HR $2,93($ IC $95 \%$ 1,29-6,66) y crecen en respuestas patológicas completas HR 0,43 (IC95\% 0,21-0,87).

Conclusión: el riesgo de recaída y de muerte se incrementa con el compromiso ganglionar masivo de 10 o más ganglios positivos y decrece en las pacientes que alcanzan respuestas patológicas completas a la quimioterapia neoadyuvante.

Palabras clave: cáncer de mama, neoadyuvancia, pronóstico.

\begin{abstract}
Objective: To evaluate overall survival and diseases - free survival in female breast cancer patients that received neoadjuvant chemotherapy treatment.

Methods: The data analysed in this study was collected between January $1^{\text {st }} 2005$ to December $31^{\text {st }} 2014$. All patients 18 and older diagnosed with breast cancer and receiving neoajuvant chemotherapy treatment were included in this study. Survival curves were created using Kaplan-Meier methods, compared using logarithmic range method and a regression analysis of proportional ranges by Cox was performed.

Results: A total of 350 patients were identified in this study with an average age of 54.8 years. The most frequent clinical stages were IIB 125 (35.71\%), IIIA 96 (27.43\%) and IIIC 58 (16.57\%). Positive estrogenic receptors were 67.14\%, 54.29\% for progesterone positive receptors and $15.43 \%$ for HER2 positive receptor. During the study they were $81(23.14 \%)$ relapses and $70(20 \%)$ deaths. Diseases free survival time decreases in massive ganglionar compromise (10 o more nodes) HR 2.96 (IC95\% 1.24-3.27) and increases in patients that achieve complete pathological responses HR 0.29 (IC 95\% 0.11-0.72). Global survival time decreases if they are 10 or more positive nodes HR 2.93 (IC95\% 1.29-6.66) and it increases in patients that achieved complete patological responses to neoadyuvant chemoterapy treatment HR 0.43 (IC95\% 0.21-0.87).
\end{abstract}

Conclusion: Relapse and death risk increases with massive ganglionar compromise (10 or more positive nodes) and decreases in patients to achieve complete pathological responses to the neoadyuvant chemotherapy treatment.

Key words: Breast cancer, neoadyuvant, prognosis. 


\section{Introducción}

Según la información de incidencias y mortalidad de Globocan del período 2018, el cáncer de mama es el primero en incidencia en mujer en el mundo, pero el segundo más frecuente después del cáncer de pulmón en ambos sexos. Es la primera causa de mortalidad por cáncer en mujeres y es la quinta causa en ambos sexos. En Colombia, el cáncer de mama ocupa el primero en incidencia con 13.380 casos nuevos; en mortalidad ocupa el cuarto puesto en ambos sexos con 3.702 casos $^{1}$.

La quimioterapia neoadyuvante es un estándar de tratamiento en cáncer de mama localmente avanzado (IIIA, IIIB, IIIC) con la finalidad de hacer posible la cirugía en estadios iniciales (IIA y IIB); se usa para permitir cirugía conservadora, evaluar quimiosensibilidad y facilitar la investigación traslacional².

El metaanálisis de Cochrane de 2007 identificó 14 estudios para un total de 5.500 mujeres con medianas de seguimiento de 18 a 124 meses; se evidenció que la quimioterapia neoadyuvante cuando alcanza respuestas patológicas completas se asocia con mejor sobrevida (HR 0,48, IC95\% 0,33-0,69) .

El estudio del MD Anderson de la Universidad de Texas evaluó los resultados de la quimioterapia neoadyuvante en las pacientes de acuerdo con las respuestas y los cinco años de sobrevida global y sobrevida libre de enfermedad, y halló que son mejores en el grupo que tiene respuestas patológicas completas, $89 \%$ y $87 \%$, que en el grupo con respuestas menores, $64 \%$ y $58 \%$, respectivamente ${ }^{4}$.

Además de la respuesta a la quimioterapia, otros factores, como el estadio clínico, el estado de los receptores hormonales y del HER2, influyen en el pronóstico de cáncer de mama que recibe tratamiento neoadyuvante ${ }^{5}$.

A pesar de estos resultados, no es claro qué magnitud del beneficio definido por incremento de respuestas patológicas completas es asociado al aumento de sobrevida global y libre de enfermedad. Los investigadores hipotetizan que la carencia de esta información puede deberse a que la población de pacientes incluida en muchos estudios es heterogénea, a la baja tasa de respuestas patológicas completas aun en brazos de tratamiento superior y a la carencia de agentes blanco efectivo en muchas de las pacientes de la población estudiada ${ }^{2-6}$.
Diversos modelos han sido desarrollados con el fin de definir mejor el pronóstico de las pacientes tratados con neoadyuvancia, entre estos está la carga residual tumoral (RBC), que tiene en cuenta el tamaño del tumor primario residual, el porcentaje de celularidad del tumor invasivo, el número de ganglios positivos y el tamaño del depósito tumoral ganglionar más grande, pero los patólogos rutinariamente no reportan esta información y la validación es limitada, así que se requieren más estudios para incorporar este modelo en la práctica clínica7-9.

Este estudio tiene como objetivo mostrar la sobrevida global y libre de enfermedad en una cohorte de mujeres con cáncer de mama tratadas con quimioterapia neoadyuvante en la clínica Oncocare en Bogotá (Colombia) durante el período 2005-2014.

\section{Métodos}

\section{Población}

Durante el período 2005-2014, se siguió una cohorte de 350 mujeres con cáncer de mama tratadas con quimioterapia neoadyuvante en la clínica Oncocare en Bogotá (Colombia). Los seguimientos se realizaron durante el tratamiento neoadyuvante: cada tres semanas se evaluaba la respuesta clínica según criterios Recist 1.0 y, al finalizar la quimioterapia, se hacía seguimiento cada 4 a 6 meses durante los cinco primeros años y luego anualmente. En los seguimientos a las mujeres, se efectuaba examen clínico y mamografía anual, y, de acuerdo con los hallazgos clínicos, se llevaban a cabo estudios complementarios para documentar recaídas de la enfermedad. Para documentar el estado vital de las mujeres que se perdieron del seguimiento, se hicieron llamadas por parte de la trabajadora social o a través de la página de web del Fosyga que determinaban ese párametro.

\section{Diseño}

Se trata de un estudio de tipo cohorte analítico bidireccional con un componente retrospectivo y prospectivo. Las pacientes que ingresaron desde el $1^{\circ}$ de enero de 2005 hasta el 31 diciembre de 2008 corresponden al componente retrospectivo; las pacientes que ingresaron a la cohorte a partir del $1^{\circ}$ de enero de 2009 hasta el 31 de julio de 2014 corresponden al componente prospectivo. 


\section{Objetivo principal}

Determinar la sobrevida global de las mujeres con cáncer de mama que reciben tratamiento de quimioterapia neoadyuvante.

\section{Objetivos secundarios}

1. Determinar la sobrevida libre de enfermedad de las mujeres con cáncer de mama que reciben tratamiento de quimioterapia neoadyuvante.

2. Establecer la asociación entre los factores pronóstico, como estado ganglionar, receptores hormonales, receptor HER2, tamaño tumoral, estadio clínico, respuestas patológicas completas y edad, con la sobrevida global de las mujeres con cáncer de mama que reciben tratamiento de quimioterapia neoadyuvante.

3. Establecer la asociación entre los factores pronóstico, como estado ganglionar, receptores hormonales, receptor HER2, tamaño tumoral, estadio clínico, respuestas patológicas completas y edad, con la sobrevida libre de enfermedad de las mujeres con cáncer de mama que reciben tratamiento de quimioterapia neoadyuvante.

\section{Resultados medidos}

Se calcularon la sobrevida libre de enfermedad, que se definió como el tiempo desde el diagnóstico hasta la primera recaída o muerte por alguna causa; y la sobrevida global, que se definió como el tiempo desde el diagnóstico hasta la muerte por cualquier causa.

Se determinó la respuesta clínica siguiendo los criterios Recist 1.0. Los criterios se han adaptado del original del manual OMS teniendo en cuenta la medición del diámetro más largo para todas las lesiones blanco ${ }^{10}$ :

- Respuesta completa: la desaparición de todas las lesiones blanco.

- Respuesta parcial: disminución de al menos un $30 \%$ en la suma de los diámetros más largos de las lesiones blanco, tomando como referencia la suma de las línea de base.

- Enfermedad progresiva: por lo menos un aumento del $20 \%$ en la suma del diámetro más largo de las lesiones o la aparición de nuevas lesiones, una o más.

- Enfermedad estable: no hay reducción suficiente para calificar como respuesta parcial ni aumento suficiente para calificar como enfermedad progresiva. Las respuestas clínicas completas se confirmaron por patología. Para respuestas completas (yPTO/Is) y (yPNO), se usó el criterio de desaparición de las lesiones invasivas del tumor primario y desaparición de la lesión ganglionar. La respuesta patológica tanto en la mama como en la axila ha sido evaluada según los criterios de Sataloff'11.

\section{Análisis estadístico}

Se realizaron análisis descriptivos usando medidas de tendencia central, ubicación y dispersión para las variables continuas; y frecuencias absolutas y relativas para las variables categóricas. Se estimaron funciones de sobrevida libre de enfermedad y global, utilizando el método no paramétrico de Kaplan-Meier, las cuales se compararon empleando la prueba de logaritmo del rango (logrank). Se estimaron razones de peligro (HR) crudas y ajustadas usando regresión de Cox; para el ajuste se utilizaron las variables con resultados significativos en la prueba de logrank. En los modelos de regresión de Cox, se verificó el supuesto de proporcionalidad; en los casos en que fue violado, la interacción entre la variable independiente y el tiempo fue introducida en el modelo. Los análisis se efectuaron a dos colas para un nivel de error tipo I de 0,05. El programa Stata 11 fue usado para el análisis.

\section{Resultados}

Las características de las mujeres con cáncer de mama que participaron en la cohorte son descritas en la tabla 1. Un total de 350 pacientes fueron identificadas en el estudio: 120 en el componente retrospectivo y 230 en el prospectivo. La edad media fue de 54,8 años. El estadio clínico más frecuente fue IIIB $125(35,71 \%)$, seguido de IIIA $96(27,43 \%)$ y IIIC 58 $(16,57 \%)$. Los receptores de estrógenos son positivos en un $67,14 \%$, los de progestágeno son positivos en un $54,29 \%$ y los receptores de HER2 son positivos en un $15,43 \%$.

En la tabla 2 se muestra la respuesta clínica obtenida en las mujeres tratadas con quimioterapia neoadyuvante. Se observa que los esquemas ACTH y ACT fueron los que obtuvieron un porcentaje mayor de respuestas patológicas completas. Las respuestas a la quimioterapia neoadyuvante fueron: respuesta 
Tabla 1. Características clínicas de la cohorte de cáncer de mama avanzado

\begin{tabular}{|c|c|}
\hline Edad promedio (SD) & $54,8(12,02)$ \\
\hline Estadio clínico & $\mathrm{n}(\%)$ \\
\hline$\| \mathrm{A}$ & $15(4,29)$ \\
\hline IIB & $56(16,00)$ \\
\hline IIIA & $96(27,43)$ \\
\hline IIIB & $125(35,71)$ \\
\hline IIIC & $58(16,57)$ \\
\hline \multicolumn{2}{|l|}{ Tipo histológico } \\
\hline Adenocarcinoma ductal & $305(87,14)$ \\
\hline Adenocarcinoma lobulillar & $22(6,29)$ \\
\hline Mixto & $3(0,86)$ \\
\hline Sin información & $20(5,71)$ \\
\hline \multicolumn{2}{|l|}{ Grado histológico } \\
\hline Grado I & $28(8,0)$ \\
\hline Grado II & $178(50,86)$ \\
\hline Grado III & $90(25,71)$ \\
\hline Sin información & $54(15,43)$ \\
\hline \multicolumn{2}{|l|}{ Estado funcional } \\
\hline ECOG 0 & $271(77,43)$ \\
\hline ECOG 1 & $79(22,57)$ \\
\hline \multicolumn{2}{|l|}{ Seguridad social } \\
\hline Contributivo & $163(46,57)$ \\
\hline Subsidiado & $187(53,43)$ \\
\hline \multicolumn{2}{|l|}{ Estado receptores } \\
\hline Receptores estrógenos & $235(67,34)$ \\
\hline Receptores progesterona & $190(54,29)$ \\
\hline Receptor HER2 & $54(15,43)$ \\
\hline \multicolumn{2}{|c|}{ Tipo quimioterapia neoadyuvante } \\
\hline$A C$ & $179(51,44)$ \\
\hline ACT & $116(33,14)$ \\
\hline $\mathrm{ACTH}$ & $41(11,71)$ \\
\hline \multicolumn{2}{|l|}{ Estado ganglionar } \\
\hline Negativos & $125(35,71)$ \\
\hline Positivos & $187(53,42)$ \\
\hline Sin información & $38(10,85)$ \\
\hline
\end{tabular}

Tabla 2. Respuesta clínica quimioterapia (Recist)

\begin{tabular}{|c|c|c|c|c|}
\hline $\begin{array}{c}\text { Tipo de quimio- } \\
\text { terapia n (\%) }\end{array}$ & Completa & Parcial & Estable & Progresión \\
\hline AC & $19(10,6)$ & $113(63,1)$ & $43(24,0)$ & $4(2,2)$ \\
\hline CMF & $2(4,8)$ & $29(70,7)$ & $8(19,5)$ & $2(4,8)$ \\
\hline ACT & $30(25,8)$ & $65(56,0)$ & $14(12,0)$ & $7(6,0)$ \\
\hline ACTH & $3(27,2)$ & $7(63,6)$ & $0(0,0)$ & $1(0,9)$ \\
\hline Total & $54(16,1)$ & $212(63,2)$ & $65(19,4)$ & $14(4,1)$ \\
\hline
\end{tabular}

AC: cilofosfamida-doxorrubicina

ACT: cilofosfamida-doxorrubicina-paclitaxel

ACTH: cilofosfamida-doxorrubicina-paclitaxel-trastuzumab

CMF: ciclofosfamida-metrotexate-fluorouracilo

objetiva en un 79,3\% (respuesta completa: $16,1 \%$ y respuesta parcial: $63,2 \%)$; las respuestas patológicas completas fueron menores que las clínicas con un $14,2 \%$, lo cual equivale al $88 \%$ de las respuestas clínicas.

La mediana del tiempo de seguimiento fue de 54,9 meses. Durante el seguimiento se observaron 81 eventos de recaída y 70 eventos de muerte.

Las funciones de sobrevida global y libre de enfermedad son ilustradas en las figuras 1 y 2 .

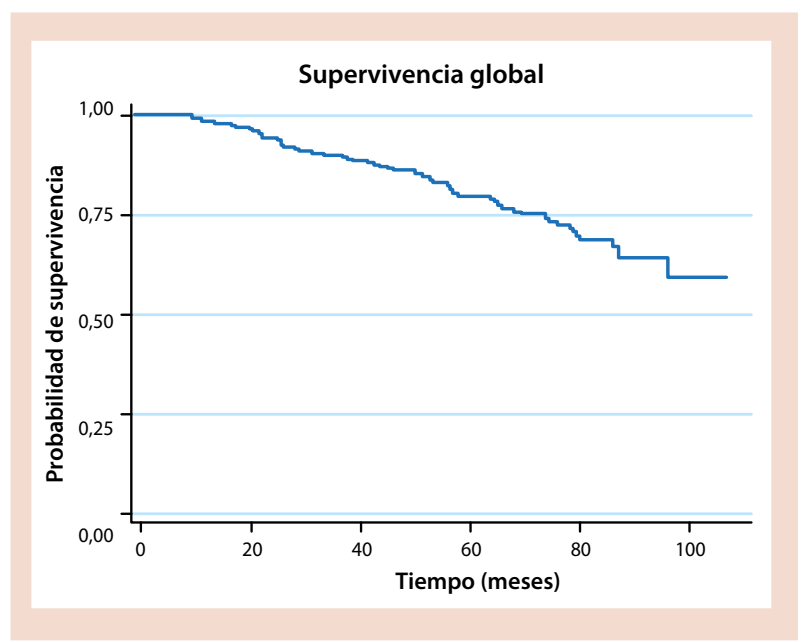

Figura 1. Supervivencia global de la cohorte de mujeres con cáncer de mama tratamiento neoadyuvante.

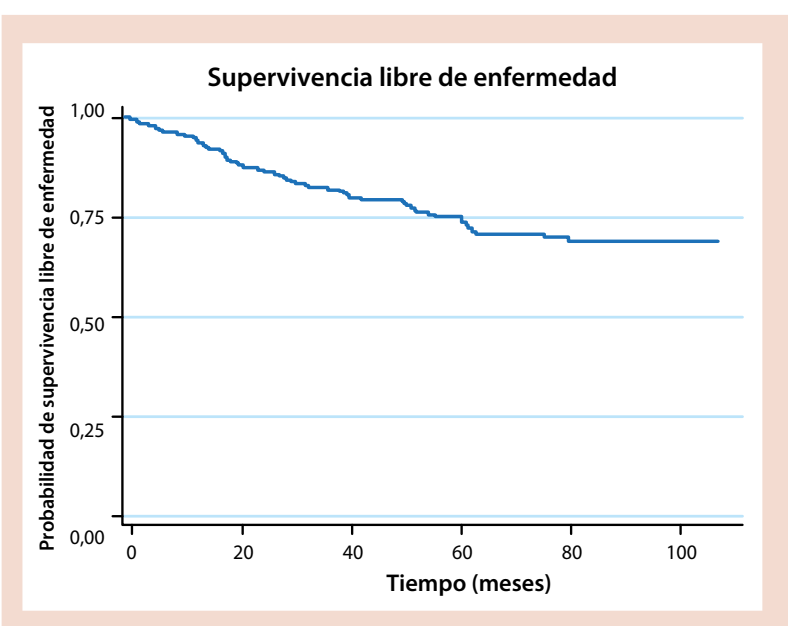

Figura 2. Supervivencia libre de enfermedad de la cohorte de mujeres con cáncer de mama tratamiento neoadyuvante.

Los resultados del modelo de regresión de Cox para la sobrevida global se exponen en la tabla 3. Los tiempos de sobrevida global se ven disminuidos por la presencia de 10 o más ganglios positivos HR 2,93 (IC95\% 1,29-6,66) y aumentan en pacientes que alcanzan respuestas patológicas completas a la quimioterapia neoadyuvante HR 0,43 (IC95\% 0,210,87 ), pero no hay asociación de las variables estadio clínico, receptor hormonal, receptor HER2, edad y tamaño tumoral con la sobrevida.

En la tabla 4, se evidencian los resultados del modelo de Cox para la sobrevida libre de enfermedad. Los tiempos de sobrevida se ven reducidos por el compromiso ganglionar masivo (10 o más ganglios positivos) HR 2,96 (IC95\% 1,24-3,27) y se 
Tabla 3. Resultados módulo de Cox para la supervivencia global

\begin{tabular}{|c|c|c|}
\hline Variable & HR crudo (IC95\%) & $\begin{array}{l}\text { HR ajustado } \\
\text { (IC95\%) }\end{array}$ \\
\hline \multicolumn{3}{|l|}{ Estadio clínico } \\
\hline Inicial & 1 & 1 \\
\hline Avanzado & $2,90(1,25-6,71)$ & $1,51(0,51-4,47)$ \\
\hline \multicolumn{3}{|l|}{ HER2+ } \\
\hline Negativo & 1 & 1 \\
\hline Positivo & $0,68(0,33-1,40)$ & $0,77(0,40-1,46)$ \\
\hline \multicolumn{3}{|l|}{ Receptor hormonal } \\
\hline Negativo & 1 & 1 \\
\hline Positivo & $0,77(0,40-1,46)$ & $0,76(0,54-1,22)$ \\
\hline \multicolumn{3}{|l|}{ Tamaño tumoral } \\
\hline Menor a 21 mm & 1 & 1 \\
\hline 21 a $50 \mathrm{~mm}$ & $1,76(0,96-3,52)$ & $1,21(0,84-1,61)$ \\
\hline Mayor a $50 \mathrm{~mm}$ & $1,82(0,97-3,56)$ & $1,19(0,88-1,82)$ \\
\hline \multicolumn{3}{|l|}{ Edad } \\
\hline 50 años o menos & 1 & 1 \\
\hline Mayor a 50 años & $0,94(0,77-1,19)$ & $1,06(0,78-1,46)$ \\
\hline \multicolumn{3}{|l|}{ Ganglios positivos } \\
\hline Ninguno & 1 & 1 \\
\hline 1 a 3 & $0,95(0,64-1,98)$ & $0,98(0,72-1,67)$ \\
\hline 4 a 9 & $0,97(0,65-1,74)$ & $1,21(0,81-2,54)$ \\
\hline 10 o más & $3,86(1,91-7,80)$ & $2,93(1,29-6,66)$ \\
\hline \multicolumn{3}{|l|}{ Respuesta* } \\
\hline No respuesta & 1 & 1 \\
\hline Respuesta completa & $0,08(0,01-0,64)$ & $0,43(0,21-0,87)$ \\
\hline
\end{tabular}
progresión, o respuesta parcial.

incrementan en pacientes que alcanzan respuestas patológicas completas HR 0,29 (IC95\% 0,11-0,72), pero no hay asociación de las variables tamaño tumoral mayor de $5 \mathrm{~cm}$, estadio clínico avanzado, receptor HER2, edad y receptores hormonales con la sobrevida.

\section{Discusión}

El objetivo del estudio fue analizar la sobrevida global y libre de enfermedad en una cohorte de mujeres con cáncer de mama diagnosticadas durante el período 2005-2014 que fueron tratadas con quimioterapia neoadyuvante. A la mediana de seguimiento de 54,9 meses, el $76,86 \%$ de las mujeres estaban libres de enfermedad y el $80 \%$ no habían muerto.

Un estudio colombiano que analiza la sobrevida global y libre de enfermedad en una cohorte de 1.328 mujeres con cáncer de mama reporta que en los estadios avanzados esta fue similar a la descrita en nuestro estudio ${ }^{12}$.

El resultado para sobrevida global a cinco años fue similar al reportado para mujeres de Noruega diagnosticadas durante el período 1999-2000,
Tabla 4. Resultados modelo de Cox para la supervivencia libre de enfermedad

\begin{tabular}{|c|c|c|}
\hline Variable & HR crudo (IC95\%) & $\begin{array}{l}\text { HR ajustado } \\
\text { (IC95\%) }\end{array}$ \\
\hline \multicolumn{3}{|l|}{ Estadio clínico } \\
\hline Inicial & 1 & 1 \\
\hline Avanzado & $2,02(0,79-5,10)$ & $3,33(0,89-3,95)$ \\
\hline \multicolumn{3}{|l|}{ HER2+ } \\
\hline Negativo & 1 & 1 \\
\hline Positivo & $1,01(0,52-1,99)$ & $0,95(0,59-1,62)$ \\
\hline \multicolumn{3}{|l|}{ Receptorhormonal } \\
\hline Negativo & 1 & 1 \\
\hline Positivo & $0,90(0,47-1,72)$ & $0,71(0,39-1,27)$ \\
\hline \multicolumn{3}{|l|}{ Tamaño tumoral } \\
\hline Menor a $21 \mathrm{~mm}$ & 1 & 1 \\
\hline 21 a 50 mm & $1,77(0,96-3,57)$ & $1,85(0,69-4,15)$ \\
\hline Mayor a 50 mm & $1,74(0,93-3,48)$ & $2,25(1,07-5,26)$ \\
\hline \multicolumn{3}{|l|}{ Edad } \\
\hline 50 años o menos & 1 & 1 \\
\hline Mayor a 50 años & $0,85(0,67-1,45)$ & $0,91(0,81-1,75)$ \\
\hline \multicolumn{3}{|l|}{ Ganglios positivos } \\
\hline Ninguno & 1 & 1 \\
\hline 1 a 3 & $0,99(0,61-1,38)$ & $0,95(0,69-1,66)$ \\
\hline 4a9 & $0,88(0,72-1,33)$ & $1,32(0,81-2,15)$ \\
\hline 10 o más & $1,44(0,98-2,12)$ & $2,96(1,24-3,27)$ \\
\hline \multicolumn{3}{|l|}{ Respuesta* } \\
\hline No respuesta & 1 & 1 \\
\hline Respuesta completa & $0,71(0,43-0,96)$ & $0,29(0,11-0,72)$ \\
\hline
\end{tabular}

el cual correspondió al $84 \%{ }^{12}$ y en los Estados Unidos al $89 \%{ }^{13}$.

El estado del receptor HER2 positivo no se asoció con riesgo para sobrevida libre de enfermedad y global, lo cual es similar a los estudios publicados en este sentido, probablemente por el efecto benéfico de la terapia blanco dirigida con trastuzumab ${ }^{14}$.

De los factores pronósticos analizados, se encontró que por el compromiso ganglionar masivo HR 2,96 (IC95\% 1,24-3,27) disminuyen la sobrevida libre de enfermedad y la supervivencia global HR 2,93 (1,29$6,66)$. Estos resultados son similares a los reportados en la literatura, en donde se encuentra que el número de ganglios positivos se asocia a tiempo de sobrevida disminuida ${ }^{15}$.

La respuesta patológica completa alcanzada con la quimioterapia neoadyuvante se relaciona con incremento en la sobrevida global HR 0,43 (IC95\% $0,21-0,87)$ y libre de enfermedad, de forma similar a varios estudios y metaanálisis que reportan resultados a largo plazo en mujeres estadio II/III que fueron tratadas con quimioterapia neoadyuvante ${ }^{2-6}$.

Otro factor de buen pronóstico descrito en la literatura es el estado del receptor hormonal ${ }^{16-19}$; en 
este estudio, no se observa relación con la sobrevida global y libre de enfermedad.

Este análisis tiene la limitación de no contar con un período de seguimiento largo (más de 100 meses), por lo cual el número de eventos puede no ser suficiente para realizar evaluaciones más detalladas de los factores pronósticos asociados a la sobrevida global y libre de enfermedad.

Se debe continuar con el seguimiento de esta cohorte de pacientes para determinar a largo plazo la sobrevida libre de enfermedad y global, los factores pronósticos asociados, y así poder tener información que permita caracterizar mejor el riesgo de las mujeres colombianas con cáncer de mama localmente avanzado, con el fin de ofrecer alternativas que mejoren el pronóstico de esta enfermedad en el país.

\section{Conclusión}

La sobrevida global y libre de enfermedad se disminuye con el compromiso ganglionar masivo (10 o más ganglios positivos) y las respuestas patológicas completas a la quimioterapia neoadyuvante se asocian con mejores sobrevidas. Los demás factores pronósticos analizados, como tamaño tumoral, receptor HER2, receptores hormonales y edad, no se relacionan con la sobrevida global y libre de enfermedad.

\section{Referencias}

1. International Agency for Research on Cancer. Globocan 2018 [internet]. [citado 2018 sep 12]. Disponible en: http://globocan. iarc.frl

2. Von Minckwitz G, Untch M, Blohmer JU, Costa SD, Eidtmann $\mathrm{H}$, Fasching PA, et al. Definition and impact of pathologic complete response on prognosis after neoadjuvant chemotherapy in various intrinsic breast cancer subtypes. J Clin Oncol. 2012;30(15):1796-804.

3. Mieog JS, Van der Hage JA, Van de Velde CJ. Preoperative chemotherapy for women with operable breast cancer. Cochrane Database Syst Rev. 2007;(2):CD005002.

4. Chen AM, Meric-Bernstam F, Hunt KK, Thames HD, Oswald MJ, Outlaw ED, et al. Breast conservation after neoadjuvant chemotherapy: the MD Anderson cancer center experience. J Clin Oncol. 2004;22(12):2303-12.

5. Kong X, Moran MS, Zhang N, Haffty B, Yang Q. Meta-analysis confirms achieving pathological complete response after neoadjuvant chemotherapy predicts favourable prognosis for breast cancer patients. Eur J Cancer. 2011;47(14):2084-90.

6. Cortazar $P$, Zhang L, Untch $M$, et al. Meta-analysis results from the Collaborative Trials in Neoadjuvant Breast Cancer (CTNeoBC). Cancer Res. 2012;72:93s.

7. Mathieu MC, Mazouni C, Kesty NC, Zhang Y, Scott V, Passeron J, et al. Breast Cancer Index predicts pathological complete response and eligibility for breast conserving surgery in breast cancer patients treated with neoadjuvant chemotherapy. Ann Oncol. 2012;23(8):2046-52.

8. Nahleh Z, Sivasubramaniam D, Dhaliwal $S$, Sundarajan $V_{\text {, }}$ Komrokji R. Residual cancer burden in locally advanced breast cancer: a superior tool. Curr Oncol. 2008;15(6):271-8.

9. Symmans WF, Peintinger $F$, Hatzis $C$, Rajan $R$, Kuerer $H$, Valero $V$, et al. Measurement of residual breast cancer burden to predict survival after neoadjuvant chemotherapy. J Clin Oncol. 2007;25(28):4414-22.

10. Therasse P, Arbuck SG, Eisenhauer EA, Wanders J, Kaplan $R S$, Rubinstein $L$, et al. New guidelines to evaluate the response to treatment in solid tumors. J Natl Cancer Inst. 2000;92(3):205-16.

11. Aranda Fl, Peiró G, Niveiro M, Seguí J. Neoadyuvancia en cáncer de mama: papel del patólogo. Rev Esp Patol. 2010;43:90-3.

12. Robledo JF, Caicedo JJ, DeAntonio R. Análisis de sobrevida en una cohorte de 1.328 pacientes con carcinoma de seno. Rev Colomb Cirugía. 2005;20(1):4-20.

13. Sant $M$, Allemani $C$, Berrino $F$, Coleman MP, Aareleid $T$, Chaplain $G$, et al. Breast carcinoma survival in Europe and the United States. Cancer. 2004;100(4):715-22.

14. Giltnane JM, Moeder CB, Camp RL, Rimm DL. Quantitative multiplexed analysis of ErbB family coexpression for primary breast cancer prognosis in a large retrospective cohort. Cancer. 2009;115(11):2400-9.

15. Guerra MR, Mendonca GA, Bustamante-Teixeira MT, Cintra JR, Carvalho LM, Magalhaes LM. [Five-year survival and prognostic factors in a cohort of breast cancer patients treated in Juiz de Fora, Minas Gerais State, Brazil]. Cad Saude Publica. 2009;25(11):2455-66

16. Mauriac L, Keshaviah A, Debled M, Mouridsen H, Forbes JF, Thürlimann B, et al. Predictors of early relapse in postmenopausal women with hormone receptor-positive breast cancer in the BIG 1-98 trial. Ann Oncol. 2007;18(5):859-67.

17. Kim J, Lee J, Chang E, Suh K, Lee C, Jee J, et al. Prognostic factors in patients with stage II/III breast cancer treated with adjuvant extension of neoadjuvant chemotherapy: a retrospective cohort study with ten-years of follow-up data. J Breast Cancer. 2011;14(1):39-45.

18. Dunnwald LK, Rossing MA, Li Cl. Hormone receptor status, tumor characteristics, and prognosis: a prospective cohort of breast cancer patients. Breast Cancer Res. 2007;9(1):R6.

19. Grann VR, Troxel AB, Zojwalla NJ, Jacobson JS, Hershman D, Neugut Al. Hormone receptor status and survival in a population-based cohort of patients with breast carcinoma. Cancer. 2005; 103(11):2241-51. 